\title{
Progenitors of supernova Ibc: a single Wolf-Rayet star as the possible progenitor of the SN Ib iPTF13bvn
}

\author{
Jose H. Groh ${ }^{1}$, Cyril Georgy ${ }^{2}$, and Sylvia Ekström ${ }^{1}$ \\ ${ }^{1}$ Geneva Observatory, Geneva University, Chemin des Maillettes 51, 1290 Sauverny, Switzerland \\ e-mail: jose.groh@unige.ch \\ 2 Astrophysics group, EPSAM, Keele University, Lennard-Jones Labs, Keele, ST5 5BG, UK \\ Received 26 July 2013 / Accepted 28 August 2013
}

ABSTRACT

\begin{abstract}
Core-collapse supernova (SN) explosions mark the end of the tumultuous life of massive stars. Determining the nature of their progenitors is a crucial step towards understanding the properties of SNe. Until recently, no progenitor has been directly detected for SN of type Ibc, which are believed to come from massive stars that lose their hydrogen envelope through stellar winds and from binary systems where the companion has stripped the $\mathrm{H}$ envelope from the primary. Here we analyze recently reported observations of iPTF13bvn, which could possibly be the first detection of a SN Ib progenitor based on pre-explosion images. Very interestingly, the recently published Geneva models of single stars can reproduce the observed photometry of the progenitor candidate and its mass-loss rate, confirming a recently proposed scenario. We find that a single WR star with initial mass in the range $31-35 M_{\odot}$ fits the observed photometry of the progenitor of iPTF13bvn. The progenitor likely has a luminosity of $\log \left(L_{\star} / L_{\odot}\right) \sim 5.55$, surface temperature $\sim 45000 \mathrm{~K}$, and mass of $\sim 10.9 M_{\odot}$ at the time of explosion. Our non-rotating $32 M_{\odot}$ model overestimates the derived radius of the progenitor, although this could likely be reconciled with a fine-tuned model of a more massive (between 40 and $50 M_{\odot}$ ), hotter, and luminous progenitor. Our models indicate a very uncertain ejecta mass of $\sim 8 M_{\odot}$, which is higher than the average of the SN Ib ejecta mass that is derived from the lightcurve $\left(2-4 M_{\odot}\right)$. This possibly high ejecta mass could produce detectable effects in the iPTF13bvn lightcurve and spectrum. If the candidate is indeed confirmed to be the progenitor, our results suggest that stars with relatively high initial masses $\left(>30 M_{\odot}\right)$ can produce visible SN explosions at their deaths and do not collapse directly to a black hole.
\end{abstract}

Key words. supernovae: general - supernovae: individual: Ib iPTF13bvn - stars: evolution - stars: Wolf-Rayet stars: winds, outflows - stars: massive

\section{Introduction}

Core-collapse supernova (CCSN) explosions mark the end of the life of stars with initial masses $\left(M_{\text {ini }}\right)$ above $\sim 8 M_{\odot}$. CCSNe are generally luminous events that are classified according to their spectrum and lightcurve (Filippenko 1997), with type II $\mathrm{SNe}$ presenting strong $\mathrm{H}$ lines in their spectrum. Conversely, type I CCSNe do not present $\mathrm{H}$ lines and are further subdivided into types Ic (without He lines) and Ib (with He lines).

Most of the observed features of SNe, such as the temporal evolution of the spectrum, absolute magnitudes, and colors, are determined by characteristics that can be traced back to properties of the progenitor, such as its mass, radius, and chemical composition. For that reason, it is thought that different kinds of $\mathrm{SNe}$ are produced by different progenitors, which in general would come from both single and binary stars.

The properties of SN progenitors can be constrained using several complementary techniques. These include direct imaging of the progenitor in pre-explosion images (e.g. Smartt 2009), hydrodynamical modeling of the lightcurve (e.g. Nomoto et al. 1993) and spectrum (e.g. Dessart et al. 2011), and analysis of the progenitor's environment (e.g. Modjaz et al. 2008), among others. To extract all the information about the progenitor contained in the observations, these techniques are often used in combination with stellar evolution models.

While the progenitors of SN II have fortuitously been directly imaged in the past decades (Smartt 2009), no detection of a SN Ibc progenitor has been obtained until recently (Smartt 2009; Eldridge et al. 2013, hereafter E13). Supernova Ibc progenitors are believed to come in part from massive stars that lose their $\mathrm{H}$ envelope due to stellar winds, becoming Wolf-Rayet (WR) stars (Maeder 1981), and in part from binary systems where the $\mathrm{H}$ envelope has been stripped by a companion (Podsiadlowski et al. 1992). However, the non-detection of WRs as SN Ibc progenitors has cast doubt on whether single WR stars could produce SN Ibc. While Smartt (2009) and E13 suggest that single WRs are bright and should have been detected in pre-explosion images, Yoon et al. (2012) suggest that single WR stars at the pre-SN stage could be very hot and faint in the optical bands. Based on detailed evolutionary and atmospheric modeling, Groh et al. (2013b; hereafter G13) confirm that $\mathrm{SN}$ Ibc progenitors from single stars are too faint to have been detected in the pre-explosion optical images of the SN Ibc sample from E13. Therefore, not detecting WR stars in SN pre-explosion images do not preclude them from being the progenitors of SN Ibc.

Recently, Cao et al. (2013; hereafter C13) report a possible detection of the progenitor of the SN Ib iPTF13bvn. The candidate source was detected within the $2 \sigma$ error of the SN astrometry and, based on its absolute magnitudes, has been suggested as a massive WR star, with a hydrostatic radius smaller than a few $R_{\odot}$ and a mass-loss rate $(\dot{M})$ of $\sim 3 \times 10^{-5} M_{\odot} \mathrm{yr}^{-1}$ (C13).

Our goal here is to investigate the nature of the possible progenitor of iPTF13bvn, and constrain the luminosity, surface temperature, spectral type, and initial mass of the progenitor. This is achieved by comparing theoretical predictions of SN progenitors, based on combined stellar evolution and atmospheric models from G13 to the observed properties of iPTF13bvn from C13. 

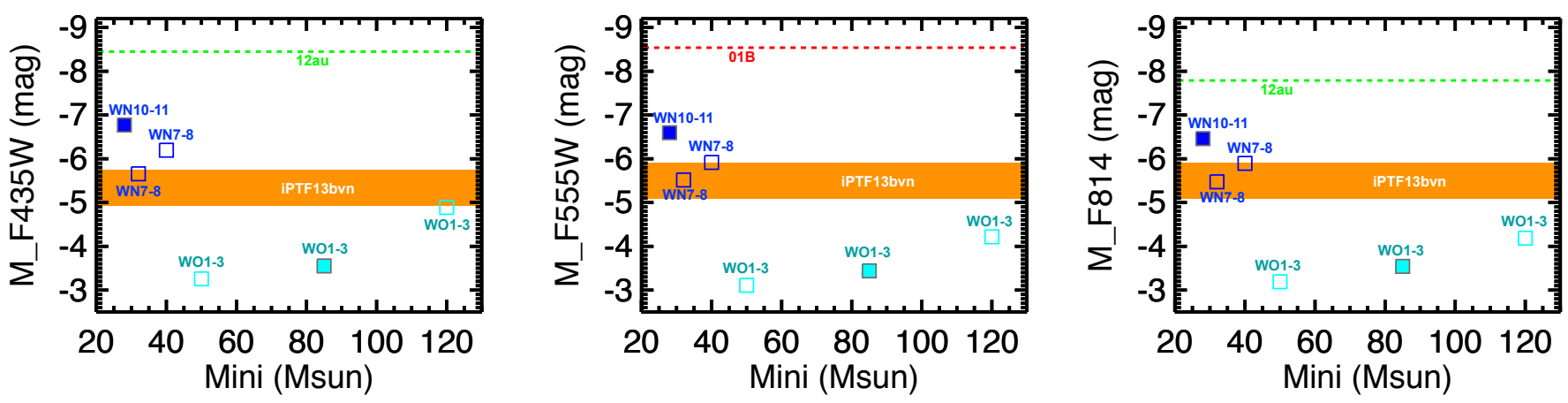

Fig. 1. Absolute magnitude of the SN Ib progenitor models as a function of $M_{\mathrm{ini}}$, in the ACS/WFC F435W (left), $F 606 W$ (middle), and $F 814 W$ filters (right). The progenitor spectral type is color coded, with cyan symbols corresponding to WOs and blue symbols to late WN stars. The evolutionary model type is coded as open (without rotation) and filled (with rotation) symbols. The orange regions correspond to the absolute magnitudes of iPTF13bvn derived from the measured photometry. The upper limits of other SN Ib progenitors (2001B and 2012au, from E13) are shown by horizontal dashed lines. The non-rotating $32 M_{\odot}$ model fits the observations of the iPTF13bvn progenitor relatively well.

\section{Stellar evolution and atmospheric models of SN progenitors}

Our strategy here is to employ our recent Geneva stellar evolution and atmospheric models of stars $M_{\text {ini }}=9-120 M_{\odot}$, computed in Groh et al. (2013a) and G13. Therefore, the models presented here are not fine-tuned to reproduce any specific observation. Below we briefly remind the reader of the main properties of the models and refer to the aforementioned papers for further details.

The evolutionary models were computed with the Geneva stellar evolution code and are presented in their vast majority in Ekström et al. (2012). A few additional models are shown in G13. The models assume solar metallicity ${ }^{1}$ and were computed both for the case of no initial rotation and for an initial rotational speed of $40 \%$ of the critical velocity on the zero-age main sequence. The output spectra are computed using the atmospheric radiative transfer code CMFGEN (Hillier \& Miller 1998), which is a state-of-the-art, full line-blanketed, non-local thermodynamical equilibrium, spherically symmetric code. As input parameters, the models computed here assume the physical conditions provided by the stellar evolutionary calculations, such as the hydrostatic radius $R_{\star}$, luminosity $L_{\star}$, mass $M_{\star}$, and surface abundances. The stellar evolution solution and the atmospheric solution are merged using the temperature structure of the envelope. A hydrostatic solution is computed for the subsonic atmosphere and is joined to a $\beta$-type wind velocity law. Our models consider wind clumping with a volume-filling factor of $f=0.1$.

Our grid of models allows one to employ, for the first time, theoretical synthetic spectra and photometry of SN progenitors that were computed self-consistently with the evolutionary calculations to analyze the observations of SN progenitors.

\section{The elusive progenitors of $\mathrm{SN} \mathrm{Ibc}$ and the case of iPTF13bvn}

The nature of the SN Ib progenitors from single stars depends on their $M_{\text {ini }}$ and rotation (G13). We do not expect that our models are able to reproduce all SN Ib progenitor observations, but only those arising from single stars. We briefly recall the main findings from G13 concerning the $\mathrm{SN}$ Ib progenitors from single stars (see their Fig. 4). Non-rotating models predict that $72 \%$ of the progenitors are late WR stars of the WN subtype

\footnotetext{
1 The host galaxy of iPTF13bvn (NGC 5806) has an oxygen abundance of $12+\log \left(N_{\mathrm{O}} / N_{\mathrm{H}}\right)=8.5$ (Smartt et al. 2009), so the use of models at solar metallicity is appropriate.
}

(WN7-8) and have $32.0 M_{\odot}<M_{\text {ini }}<45.0 M_{\odot}$. The remaining $28 \%$ of the SN Ib progenitors are WR stars of the WO subtype (WO1-3) with 45.0 $M_{\odot}<M_{\text {ini }}<52.2 M_{\odot}$ or $106.4 M_{\odot}<M_{\text {ini }}<$ $120.0 M_{\odot}$. In this context, the non-rotating models are representative of stars that are born as slow rotators. Rotating models with $v_{\text {rot }} / v_{\text {crit }}=0.4$ indicate that $96 \%$ of the $\mathrm{SN} \mathrm{Ib}$ progenitors are WN 10-11 stars $\left(25.0 M_{\odot}<M_{\text {ini }}<30.1 M_{\odot}\right)$, while $4 \%$ are WO $1-3$ stars $\left(82.0 M_{\odot}<M_{\text {ini }}<88.7 M_{\odot}\right)$. These mass ranges assume chemical abundance criteria to determine the $\mathrm{SN}$ type, with $\mathrm{SN} \mathrm{Ib}$ having no $\mathrm{H}$ and more than $0.6 M_{\odot}$ of $\mathrm{He}$ in the ejecta, and SN Ic having no $\mathrm{H}$ and less than $0.6 M_{\odot}$ of He. As discussed in, say, Georgy et al. (2012), the mass ranges quoted above are uncertain.

Our models produce several quantities that can be compared to data derived from the observations of SN progenitors, allowing one to constrain the nature of the progenitor. We argue that absolute magnitudes are the most direct property that can be employed in the comparison, since they are obtained from the observations of SN progenitors in an almost model-independent way.

Figure 1 presents the absolute magnitudes of our SN Ib progenitors as a function of $M_{\text {ini }}$. These broadband absolute magnitudes are computed by convolving the high-resolution spectrum of the progenitor with a given filter bandpass. As such, they include both the contributions of spectral lines and continuum, since both are probed by the observed broadband photometry. In the present case, we use the same filters in which the possible iPTF13bvn progenitor was detected (Hubble Space Telescope/Advanced Camera for Surveys, HST/ACS). This illustrates one of the strengths of our approach, since no filter transformations between different photometric systems are needed.

Our progenitor models can be divided into two main groups: relatively bright $\left(M_{555 \mathrm{~W}}=-5.5\right.$ to $\left.-6.2 \mathrm{mag}\right)$ massive WNLs, and relatively faint $\left(M_{555 \mathrm{~W}}=-3.1\right.$ to $\left.-4.1 \mathrm{mag}\right)$, very massive WOs. For other well-classified SN Ib, such as SN 2001B and SN 2012au, all models lie below the detection threshold, in agreement with a non-detection in the pre-explosion images (G13). To avoid errors due to filter transformations, we decided to use the original HST/ACS photometry of iPTF13bvn from $\mathrm{C} 13$ to obtain the absolute magnitudes in these filters, instead of using the absolute magnitudes in $B V I$ filters provided by these authors. They measured $m_{435 \mathrm{~W}}=26.7 \pm 0.2 \mathrm{mag}$, $m_{555 W}=26.5 \pm 0.2 \mathrm{mag}$, and $m_{814 W}=26.4 \pm 0.2 \mathrm{mag}$. We also assume the same distance $(d=22.5 \mathrm{Mpc}$; Tully et al. 2009), and reddening (local extinction $E(B-V)=0.0437$, 

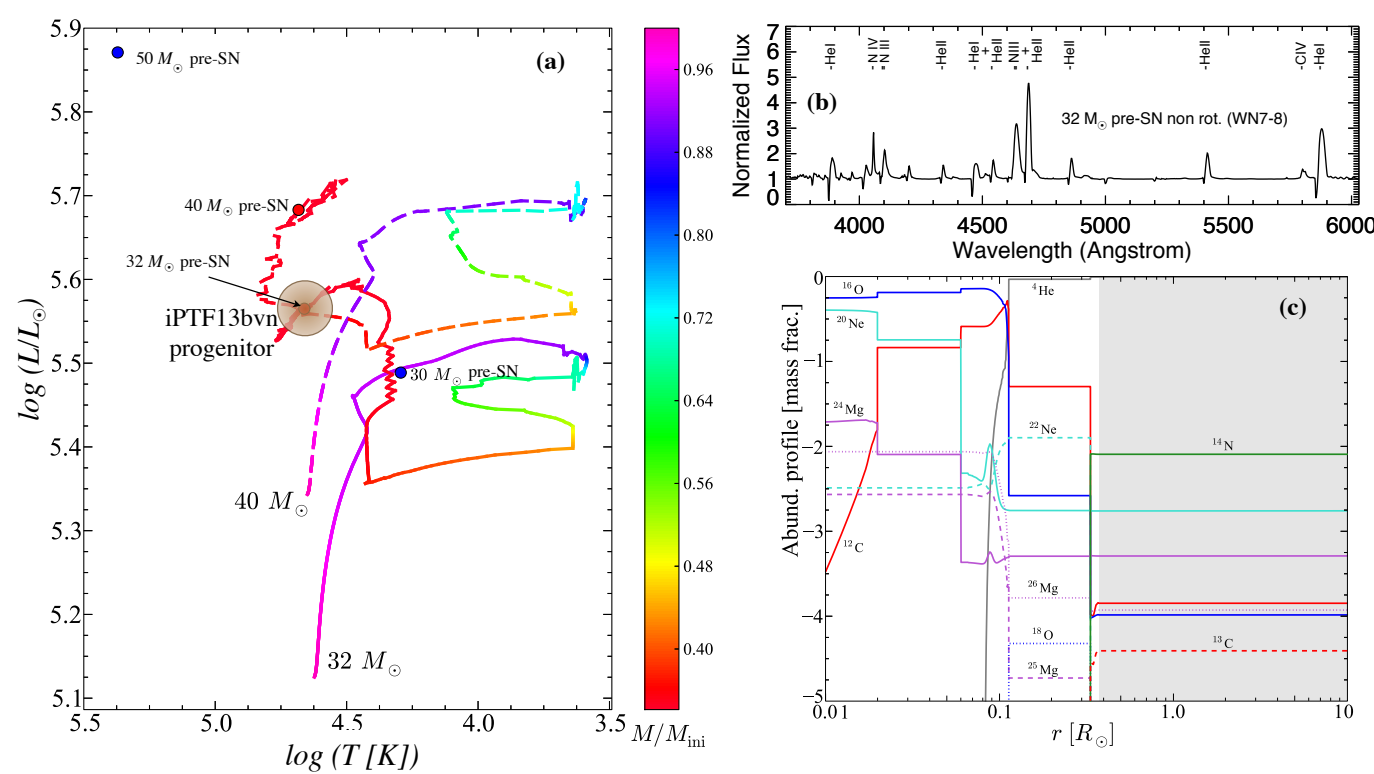

Fig. 2. a) Hertzprung-Russell diagram showing the evolutionary tracks of non-rotating $32 M_{\odot}$ (solid) and $40 M_{\odot}$ models (dashed), with the red circles representing their endpoints. The location of the possible progenitor of iPTF13bvn is depicted by the brown region, whose size does not correspond to a formal error analysis and is merely indicative of the possible location of the progenitor. The color code refers to the mass at each timestep divided by $M_{\text {ini }}$. The endpoints of the non-rotating 30 and $50 M_{\odot}$ models are also shown (blue circles). b) Continuum-normalized optical spectrum of the $32 M_{\odot}$ model at the pre-SN stage, showing it is a typical WN7-8 star (G13). The strongest features are identified. c) Chemical structure of our $32 M_{\odot}$ model at the end of the core carbon-burning stage, when the model has $10.9 M_{\odot}$. The variations in the mass fraction of different species is shown as a function of the radius, with the surface of the star being to the right, at $9.69 R_{\odot}$, and the center to the left at $0.0 R_{\odot}$.

foreground extinction $E(B-V)=0.0278$, and $\left.R_{V}=3.1\right)$ estimated by $\mathrm{C} 13$. We thus find that the possible progenitor has $M_{435 W}=-5.3 \pm 0.4 \mathrm{mag}, M_{555 \mathrm{~W}}=-5.5 \pm 0.4 \mathrm{mag}$, and $M_{814 W}=-5.5 \pm 0.4 \mathrm{mag}$.

We find that some of the non-rotating WNL SN Ib progenitor models are in excellent agreement with the observed photometry (Fig. 1). This is remarkable given that the models have not been fine tuned. For instance, the non-rotating model with $M_{\text {ini }}=32 M_{\odot}$ has $M_{435 \mathrm{~W}}=-5.66 \mathrm{mag}, M_{555 \mathrm{~W}}=$ -5.52 mag, and $M_{814 W}=-5.47 \mathrm{mag}$. The non-rotating $40 M_{\odot}$ model is brighter than the observations in the $F 435 \mathrm{~W}$. The $50 M_{\odot}$ model is too faint in all bands in which the progenitor was detected. $\mathrm{SN}$ Ib progenitors with $M_{\text {ini }}>50 M_{\odot}$ are WO stars (cyan) and are typically at least 1 mag fainter than the observations. SN Ib progenitors with $M_{\mathrm{ini}}=28 M_{\odot}$ are too bright and can also be discarded. SN progenitors with $M_{\text {ini }}<25 M_{\odot}$ do not produce SN Ib (Georgy et al. 2012). Thus, while our non-rotating $32 M_{\odot}$ model fits the observed photometry of the possible progenitor of iPTF13bvn best, we cannot discard that a fine-tuned non-rotating model between 40-50 $M_{\odot}$ (or a rotating model between 28 and $32 M_{\odot}$ ) could also fit the data (Fig. 1). We note that the absolute magnitudes of $\mathrm{SN}$ Ib progenitors are strongly dependent on $M_{\text {ini }}$ (Fig. 1), which is the reason $M_{\text {ini }}$ is well constrained.

Therefore, if the detected star is indeed the progenitor of the SN Ib iPTF13bvn, our models indicate that the progenitor is consistent with a single WR star that had $M_{\text {ini }} \sim 31-35 M_{\odot}$. Figure $2 \mathrm{a}$ shows the evolutionary tracks of the non-rotating 32 and $40 M_{\odot}$ models in the Hertzprung-Russell diagram. The progenitor of iPTF13bvn would have a final mass of $10.9 M_{\odot}$, surface temperature of $T_{\star} \sim 45000 \mathrm{~K}$ (computed at a Rosseland optical depth of $\tau_{\text {Ross }}=20$ ), effective temperature of $\sim 40000 \mathrm{~K}$ (computed at $\left.\tau_{\text {Ross }}=2 / 3\right)$, and $\log \left(L_{\star} / L_{\odot}\right) \sim 5.55$. According to our models, the progenitor would have a spectral type of WN $7-8 \mathrm{o}$, with the optical spectrum characterized by broad, strong emission lines of He II, He I, N III, and N IV (Fig. 2b).
Our models also predict other theoretical quantities that can be compared to those derived from the observations. We stress here that unlike the progenitor photometry, these other quantities are obtained from the observations by modeling the SN lightcurve and/or spectrum. In the case of iPTF13bvn, there are two other physical properties that have been determined (see $\mathrm{C} 13$ ), namely the progenitor hydrostatic radius (less than a few $R_{\odot}$ ) and $\dot{M} \sim 3 \times 10^{-5} M_{\odot} \mathrm{yr}^{-1}$. Regarding $\dot{M}$, our non-rotating $32 M_{\odot}$ model predicts that the SN progenitor will have $\dot{M}=2.9 \times 10^{-5} M_{\odot} \mathrm{yr}^{-1}$, in excellent agreement with the value derived from the observations. The hydrostatic radius of this model, however, is $9.65 R_{\odot}$, which seems to be larger than what is allowed from the lightcurve modeling ( $<$ few $R_{\odot} ; \mathrm{C} 13$ ). A fine-tuned model with $M_{\text {ini }}$ between 40 and $50 M_{\odot}$ could probably yield a lower value for the hydrostatic radius and still fit the observed photometry and mass-loss rate.

The chemical abundances and total amount of mass in the $\mathrm{SN}$ ejecta $\left(M_{\mathrm{ej}}\right)$ also provide constraints on the nature and chemical structure of the progenitor star itself. Figure $2 \mathrm{c}$ presents the chemical structure of the $32 M_{\odot}$ at the pre-SN stage, which we suggest is similar to the progenitor of iPTF13bvn. This model has no $\mathrm{H}$ left because of mass loss due to stellar winds, and the CO core has $8.9 M_{\odot}$. As of yet, no determination of $M_{\mathrm{ej}}$ of iPTF13bvn exists in the literature. Our $32 M_{\odot}$ model predicts $M_{\text {ej }}=7.89 M_{\odot}$, of which $2.89 M_{\odot}$ are composed of He (Georgy et al. 2012). We note here that while our models are able to provide a good estimate for the amount of He in the ejecta, $M_{\mathrm{ej}}$ may be quite uncertain due to difficulties in estimating the remnant and fallback mass. Based on 1D hydrodynamical models, Ugliano et al. (2012) suggest a remnant mass of $\sim 2.0 M_{\odot}$ for $32 M_{\odot}$ stars, implying $M_{\mathrm{ej}} \sim 9.0 M_{\odot}$. Although uncertain, this value of $M_{\mathrm{ej}}$ is much higher than the average $M_{\mathrm{ej}}$ of SN Ib that is usually derived from the SN lightcurve ( $\sim 2-4 M_{\odot}$; Drout et al. 2011; Cano 2013). This would imply that the high $M_{\text {ej }}$ could have detectable effects on the SN lightcurve and spectrum, making iPTF13bvn a peculiar SN Ib. Because the high luminosity of 
the candidate progenitor implies a high mass, a large $M_{\mathrm{ej}}$ would be inferred independently of whether the progenitor followed a single or a binary evolution scenario.

\section{What if the candidate star is not the progenitor of iPTF13bvn?}

As discussed by $\mathrm{C} 13$, imaging of the $\mathrm{SN}$ site in a few years time is needed to confirm that the candidate star is indeed the progenitor of iPTF13bvn. We now assume that the source detected close to the $\mathrm{SN}$ position is not the progenitor of iPTF13bvn and discuss the possible scenarios.

First, a more massive WR of the WO spectral type, with $M_{\text {ini }}=50$ to $120 M_{\odot}$, could be undetectable in the preexplosion images (Fig. 1). Assuming that the putative undetected progenitor has similar amounts of reddening as the one towards the candidate, these WOs would appear with $m_{435 \mathrm{~W}}=27.5$ to $28.9 \mathrm{mag}, m_{555 \mathrm{~W}}=27.8$ to $28.9 \mathrm{mag}$, and $m_{814 \mathrm{~W}}=27.7$ to $28.7 \mathrm{mag}$ at the distance of iPTF13bvn. They would most certainly be fainter than the magnitude limit of the pre-explosion observations, unless the latter are unusually deep. Since the specific values of the magnitude limits in the different bands are not available to us, we cannot gauge the likelihood of this scenario.

Second, we cannot leave out the possibility that a reddened WN star similar to the one discussed above, with $M_{\text {ini }}=32 M_{\odot}$, is the actual progenitor. A reddening $\sim 1-2$ mag greater than the local reddening of $E(B-V)=0.0437$ estimated by $C 13$, perhaps because of the presence of circumstellar material, would make the WN star undetectable. In this case, the progenitor would have the same nature as we suggested in Sect. 3.

Third, a low-mass, faint WR star formed via binary evolution could be the progenitor, as expected from binary models (e.g., Podsiadlowski et al. 1992). These progenitors would in principle be much fainter (because they have low mass) than the detection limit of the pre-explosion images. For instance, low-mass binary stars known as V Sagittae stars (Steiner \& Oliveira 2005), such as HD 45166 (Groh et al. 2008), have been suggested as SN Ibc progenitors (E13). As shown by Groh et al. (2008), these stars have relatively low luminosities $\left(\log \left(L_{\star} / L_{\odot}\right)=3.75\right)$ and high effective temperatures $\left(T_{\text {eff }}=50000 \mathrm{~K}\right)$, so they would be undetectable in the pre-explosion images. However, it would be challenging to reconcile the $\dot{M}$ values found for the progenitor of iPTF13bvn $\left(\sim 3 \times 10^{-5} M_{\odot} \mathrm{yr}^{-1}\right)$ with the values found for lowmass, WR-like stars that had their envelope stripped by a companion such as V Sag stars. They have $\dot{M} \sim 2 \times 10^{-7} M_{\odot} \mathrm{yr}^{-1}$ (Groh et al. 2008), which is about two orders of magnitude lower than is inferred for the progenitor of iPTF13bvn. In addition, the companion of these stars are cool, late B-type stars with a comparably low $\dot{M}$, so the companion could not be responsible for the relatively high $\dot{M}$ inferred for the progenitor.

\section{Prospects for detecting SN Ibc progenitors}

We have shown that the photometry of the candidate progenitor of the SN Ib iPTF13bvn is consistent with that of a single WR star at the pre-SN stage, confirming the results from C13. Here, we went one step further and estimated that the progenitor has a WN 7-8 spectral type and $M_{\text {ini }} \sim 31-35 M_{\odot}$. Since SN Ib comprises $35 \%$ of the total sample of SN Ibc, one may wonder to what extent it is likely that a SN Ib progenitor has possibly been detected, while many SN Ic progenitors remain undetected in pre-explosion images. Our models indicate that most of the SN Ib progenitors (70-96\%) are brighter than the SN Ic progenitors (G13). This is because the majority of SN Ib progenitors are not as hot as the SN Ic progenitors, so the earlier ones have more flux in the optical bands. Because of that, while SN Ibs are rarer than $\mathrm{SN}$ Ics, it is more likely that a $\mathrm{SN} \mathrm{Ib}$ progenitor is detected in pre-explosion images, and SN Ib progenitors will likely dominate future samples of directly detected progenitors of SN Ibc.

We can also estimate the maximum distance up to which a $\mathrm{SN}$ Ib progenitor coming from single-star evolution would be directly detected in pre-explosion images. This depends on the absolute magnitude and reddening of the progenitor in the filter of the pre-explosion image and on the magnitude limit of the image. For this purpose, we use the absolute magnitudes from Fig. 1 and assume a magnitude limit of $26.7 \mathrm{mag}$ in $F 435 \mathrm{~W}$ and low reddening (similar to the one towards the progenitor of iPTF13bvn). Since the brighter SN Ib progenitor according to out models is the one from the rotating $28 M_{\odot}$ model, we find that SN Ib progenitors could be detected up to $42 \mathrm{Mpc}$.

It is interesting to compare this maximum distance for detecting $\mathrm{SN}$ Ib progenitor with the one derived for single $\mathrm{SN}$ Ic progenitor models. For the same $F 435 \mathrm{~W}$ filter, magnitude limit, and reddening, a value of $8.4 \mathrm{Mpc}$ is obtained (G13). Thus, SN Ib progenitors should be detected up to much greater distances than SN Ic progenitors. In addition, for distances over a certain threshold ( $8.4 \mathrm{Mpc}$ given the assumptions above), the sample of directly detected SN progenitors will be incomplete, with the majority of the sources being SN II and a few being SN Ib.

The possible detection of a $\mathrm{SN}$ Ib progenitor is also extremely interesting since it indicates that a very massive progenitor $\left(M_{\mathrm{ini}}>30 M_{\odot}\right)$ can still produce a visible SN event. If a black hole $(\mathrm{BH})$ has been produced, then this would also indicate that a $\mathrm{BH}$ formation can sometimes be linked to a visible $\mathrm{SN}$ event. Of course, this last conclusion is quite speculative, since it is fairly difficult to know if the iPTF13bvn remnant is a BH or NS. However, we cannot discard that this question may be answered in the future, and efforts towards that goal are warranted.

Acknowledgements. We thank Georges Meynet for many interesting discussions and comments, and John Hillier for making CMFGEN available. JHG is supported by an Ambizione Fellowship of the Swiss National Science Foundation. CG acknowledges support from EU- FP7-ERC-2012-St Grant 306901.

\section{References}

Cano, Z. 2013, MNRAS, 1767

Cao, Y., Kasliwal, M. M., Arcavi, I., et al. 2013, ApJ, 775, L7

Dessart, L., Hillier, D. J., Livne, E., et al. 2011, MNRAS, 414, 2985

Drout, M. R., Soderberg, A. M., Gal-Yam, A., et al. 2011, ApJ, 741, 97

Ekström, S., Georgy, C., Eggenberger, P., et al. 2012, A\&A, 537, A146

Eldridge, J. J., Fraser, M., Smartt, S. J., Maund, J. R., \& Crockett, R. M. 2013, MNRAS, submitted [arXiv: 1301.1975]

Filippenko, A. V. 1997, ARA\&A, 35, 309

Georgy, C., Ekström, S., Meynet, G., et al. 2012, A\&A, 542, A29

Groh, J. H., Oliveira, A. S., \& Steiner, J. E. 2008, A\&A, 485, 245

Groh, J. H., Meynet, G., \& Ekström, S. 2013a, A\&A, 550, L7

Groh, J. H., Meynet, G., Georgy, C., \& Ekstrom, S. 2013b, A\&A, in press, DOI: 10.1051/0004-6361/201321906

Hillier, D. J., \& Miller, D. L. 1998, ApJ, 496, 407

Maeder, A. 1981, A\&A, 99, 97

Modjaz, M., Kewley, L., Kirshner, R. P., et al. 2008, AJ, 135, 1136

Nomoto, K., Suzuki, T., Shigeyama, T., et al. 1993, Nature, 364, 507

Podsiadlowski, P., Joss, P. C., \& Hsu, J. J. L. 1992, ApJ, 391, 246

Smartt, S. J. 2009, ARA\&A, 47, 63

Smartt, S. J., Eldridge, J. J., Crockett, R. M., \& Maund, J. R. 2009, MNRAS, 395, 1409

Steiner, J. E., \& Oliveira, A. S. 2005, A\&A, 444, 895

Tully, R. B., Rizzi, L., Shaya, E. J., et al. 2009, AJ, 138, 323

Ugliano, M., Janka, H.-T., Marek, A., \& Arcones, A. 2012, ApJ, 757, 69

Yoon, S.-C., Gräfener, G., Vink, J. S., Kozyreva, A., \& Izzard, R. G. 2012, A\&A, 544, L11 